Rapid Reviews COVID-19

Review 2: "Evidence for

increased breakthrough

rates of SARS-CoV-2

variants of concern in

BNT162b2 mRNA

\title{
vaccinated individuals"
}

Konstantin Chumakov ${ }^{1}$

1Office of Vaccines Research and Review, U.S. Food and Drug Administration

Published on: Apr 15, 2021

DOI: 10.1162/2e3983f5.bf467772

License: Creative Commons Attribution 4.0 International License (CC-BY 4.0). 


\section{RR:C19 Evidence Scale rating by reviewer:}

- Potentially informative. The main claims made are not strongly justified by the methods and data, but may yield some insight. The results and conclusions of the study may resemble those from the hypothetical ideal study, but there is substantial room for doubt. Decision-makers should consider this evidence only with a thorough understanding of its weaknesses, alongside other evidence and theory. Decisionmakers should not consider this actionable, unless the weaknesses are clearly understood and there is other theory and evidence to further support it.

$* * * * * * * * * * * * * * * * * * * * * * * * * * * * * * * * * * * * * * *$

\section{Review:}

The issue of emerging SARS-CoV2 variants of concern (VOC) and the ability of vaccineinduced immunity to protect against them is currently one of the most important in dealing with the pandemic. The answer will drive public health policy and future vaccine development efforts. In vitro studies suggested that some of the VOCs (e.g. B.1.1.7) are equally sensitive to neutralization with antibodies against the original strains and vaccine-induced antibodies, while neutralization of others (e.g. B.1.351 and P1) required higher concentrations of antibodies. This observation suggested that they could partially escape vaccine-induced immunity, reducing vaccine efficacy. To date, there is no clear clinical evidence one way or the other, this is why this preprint attracted significant attention.

Unfortunately, close examination of the evidence does not fully support the claims. At the time of the study, the prevalent strain in Israel was B.1.1.7, while the "wild type" rapidly declined and B.1.357 was first increasing and then went down again. Therefore, if the "matching" of cases in vaccine recipients and controls was done without making sure that they are strictly contemporary, the conclusions could be inaccurate. It is safe to assume that there were many more cases in the unvaccinated cohort, and the "matching" could be done more than once, to see if the results will hold if different controls are used. This would increase confidence in the conclusions.

Another aspect of this manuscript that needs improvement is that it is written in a rather convoluted way and contains statements that can mislead readers. On page 2 , the authors claim that the goal of the study was to see if "BNT162b2 vaccinees were more likely to become infected with B.1.1.7 or B.1.351 compared with unvaccinated 
individuals." This can be interpreted to mean that instead of protecting, vaccination increases the chances of infection with VOCs, while they probably wanted to say that vaccination protects disproportionately better from wild-type virus than VOCs.

There is also some awkward language. How can one "collect RNA from the PCR samples"? Throughout the manuscript, they use the words "fully effective" and "partially effective," which is misleading. It would be better to simply say one-dose and two-dose vaccination regimen.

In conclusion, the results are consistent with predictions that could be made from previous neutralization data. It is possible that B.1.357 can be somewhat more resistant to vaccine-induced immunity, but this manuscript cannot be cited as the unequivocal evidence of this. At best, the results cannot disprove the hypothesis. I would send the manuscript back for major revisions. 